\title{
ON OPERATOR DEFINED BY DOUBLE ZETA FUNCTIONS
}

\author{
RABHA W. IBRAHIM AND MASLINA DARUS
}

\begin{abstract}
The aim of this paper is to introduce an operator defined by generalized double zeta function involving the Riemann, Hurwitz, Hurwitz-Lerch and Barnes double zeta functions for analytic functions in the unit disc. Certain new subclasses of $\mathscr{A}$ using this operator are suggested. Some interesting properties of these classes are studied.
\end{abstract}

\section{Introduction}

In the recent years, there has been an increasing interest in problems involving evaluations of various families of series associated with the Riemann zeta function and Hurwitz zeta function and their extensions and generalizations such as the Hurwitz-Lerch zeta function. These functions arise naturally in many branches of analytic function theory and algebraic number theory, and their studies have numerous important applications in mathematics and physics (see [1, 2]]).

The Hurwitz zeta function is defined by

$$
\zeta(x, a)=\sum_{n=0}^{\infty}(a+n)^{-x},(a \neq\{0,-1,-2, . .\}, \Re(x)>1),
$$

which is a generalization of the Riemann zeta function

$$
\zeta(x)=\sum_{n=0}^{\infty} n^{-x},(\Re(x)>1) .
$$

As a generalization of both Riemann and Hurwitz zeta functions, the so-called Hurwitz-Lerch zeta function is defined by [3]

$$
\Phi(y, x, a)=\sum_{n=0}^{\infty} \frac{y^{n}}{(a+n)^{x}}, \quad(a \in \mathbb{C} \backslash\{0,-1,-2, . .\}, \Re(x)>1,|y|<1) .
$$

Corresponding author: Maslina Darus.

2000 Mathematics Subject Classification. 33C20, 30 C45.

Key words and phrases. Hurwitz-Lerch Zeta function, analytic function, hypergeometric functions. 
It is clear that $\Phi$ is an analytic function in both variables $x$ and $y$ in a suitable region and it reduces to the ordinary Lerch zeta function when $y=2 \pi i \lambda$. Moreover, $\Phi$ yields the following known result [3]:

$$
\Phi(y, 1, a)=a_{2}^{-1} F_{1}(a, 1 ; a+1, y),
$$

where ${ }_{2} F_{1}$ is the Gaussian hypergeometric function. Also we recall a further generalization of the Hurwitz-Lerch zeta function $\Phi_{\mu}(y, x, a)$ in the form [4]:

$$
\begin{gathered}
\Phi_{\mu}(y, x, a)=\sum_{n=0}^{\infty} \frac{(\mu)_{n} y^{n}}{n !(a+n)^{x}}, \\
(a \in \mathbb{C} \backslash\{0,-1,-2, . .\}, \Re(x)>1,|y|<1, \mu \in \mathbb{C})
\end{gathered}
$$

where $(\mu)_{n}=\frac{\Gamma(n+\mu)}{\Gamma(\mu)}=\mu(\mu+1) \cdots(\mu+n-1)$ denotes the Pochhammer symbol.

Recall that many interesting properties and characteristics of the Hurwitz-Lerch zeta function $\Phi_{\mu}(z, y, a)$ can be found in the recent investigations by, for example, Choi and Srivastava [5], Ferreira and Lo'pez [6], Garg et al. [7], Lin and Srivastava [8], Lin et al. [9] and Luo and Srivastava [10].

The double zeta function of Barnes [11] is defined by

$$
\zeta(x, a, v)=\sum_{n=0}^{\infty} \sum_{m=0}^{\infty}(m+a+v n)^{-x},
$$

where $a \neq 0$ and $v$ is a non-zero complex number with $|\arg (v)|<\pi$. Combining (1-3), BinSaad [12] posed a generalized double zeta function of the form

$$
\zeta_{v}^{\mu}(z, y, x, a)=\sum_{n=0}^{\infty}(\mu)_{n} \Phi(y, x, a+v n) \frac{z^{n}}{n !},
$$

where $|y|<1,|z|<1 ; \mu \in \mathbb{C} \backslash\{0,-1,-2, \ldots\}, v \in \mathbb{C} \backslash\{0\} ; a \in \mathbb{C} \backslash\{-(m+v n)\},\{n, m\} \in \mathbb{N} \cup\{0\}$ and $\Phi$ is the Hurwitz-Lerch zeta function defined by (1).

Let $\mathscr{H}$ be the class of analytic functions in $U:=\{z \in \mathbb{C}:|z|<1\}$ and $\mathscr{H}[a, n]$ be the subclass of $\mathscr{H}$ consisting of functions of the form $f(z)=a+a_{n} z^{n}+a_{n+1} z^{n+1}+\ldots$. Let $\mathscr{A}$ be the subclass of $\mathscr{H}$ consisting functions normalized by $f(0)=0$ and $f^{\prime}(0)=1$. For functions $f=z+\sum_{n=2}^{\infty} a_{n} z^{n}$ and $g=z+\sum_{n=2}^{\infty} b_{n} z^{n} \in U$, the Hadamard product or the convolution product is defined by

$$
f(z) * g(z)=z+\sum_{n=2}^{\infty} a_{n} b_{n} z^{n}, \quad(z \in U) .
$$

Using the Hadamard product of incomplete beta function which is related to Gauss hypergeometric function $\phi(a, c, z):=z_{2} F_{1}(1, a ; c, z)$, Carlson and Shaffer defined a linear operator as in [13]. Also, by using the Hadamard product of generalized hypergeometric function 
$z_{2} F_{1}(a, b ; c, z)$, Noor [14] introduced a linear operator as follows:

$$
z_{2} F_{1}(a, b ; c, z) *\left(z_{2} F_{1}(a, b ; c, z)\right)^{(-1)}=\frac{z}{(1-z)^{\lambda+1}},(\lambda>-1)
$$

implies

$$
I_{\lambda}(a, b, c) f(z)=\left(z_{2} F_{1}(a, b ; c, z)\right)^{(-1)} * f(z),(f \in \mathscr{A}) .
$$

In this work, by using the Hadamard product or the convolution product of generalized HurwitzLerch zeta function given by (4), a function is defined as follows:

$$
\Psi_{n}(y, x, a):=\frac{\Phi(y, x, a+v n)}{\Phi(y, x, a)},(n \in \mathbb{N} \cup\{0\}) .
$$

It is clear that $\Psi_{0}(y, x, a)=1$. Now consider the function

$$
\Upsilon_{\mu}(z, y, x, a)=\sum_{n=0}^{\infty} \frac{(\mu)_{n}}{n !} \Psi_{n}(y, x, a) z^{n},
$$

implies

$$
z \Upsilon_{\mu}(z, y, x, a)=z+\sum_{n=2}^{\infty} \frac{(\mu)_{n-1}}{(n-1) !} \Psi_{n-1}(y, x, a) z^{n}
$$

Thus

$$
\begin{aligned}
z \Upsilon_{\mu}(z, y, x, a) *\left(z \Upsilon_{\mu}(z, y, x, a)\right)^{(-1)} & =\frac{z}{(1-z)^{\lambda}},(\lambda>-1) \\
& =z+\sum_{n=2}^{\infty} \frac{(\lambda)_{n-1}}{(n-1) !} z^{n}
\end{aligned}
$$

poses a linear operator

$$
\begin{aligned}
I_{\mu}^{\lambda}(z, y, x, a) f(z) & =\left(z \Upsilon_{\mu}(z, y, x, a)\right)^{(-1)} * f(z),(f \in \mathscr{A}) \\
& =z+\sum_{n=2}^{\infty} \frac{(\lambda)_{n-1}}{(\mu)_{n-1} \Psi_{n-1}(y, x, a)} a_{n} z^{n},
\end{aligned}
$$

where $|y|<1,|z|<1 ; \mu \in \mathbb{C} \backslash\{0,-1,-2, \ldots\}, v \in \mathbb{C} \backslash\{0\} ; a \in \mathbb{C} \backslash\{-(m+v n)\},\{n, m\} \in \mathbb{N} \cup\{0\}$ and $\Psi$ is defined in (6). It is clear that $I_{\mu}^{\lambda}(z, y, x, a) f(z) \in \mathscr{A}$.

By using properties of the Pochhammer 's symbol, we pose

Lemma 1.1. For $f \in \mathscr{A},|y|<1,|z|<1 ; \mu \in \mathbb{C} \backslash\{0,-1,-2, \ldots\} ; a \in \mathbb{C} \backslash\{-(m+v n)\},\{n, m\} \in \mathbb{N} \cup\{0\}$ then

$$
z\left[I_{\mu}^{\lambda}(z, y, x, a) f(z)\right]^{\prime}=\lambda I_{\mu}^{\lambda+1}(z, y, x, a) f(z)-(\lambda-1) I_{\mu}^{\lambda}(z, y, x, a) f(z) .
$$

For analytic functions $f$ and $g$ in $U$, we say that $f$ is subordinate to $g$, written by $f<g$, if there exists an analytic function $w$ with $w(0)=0$ and $|w(z)|<1(z \in U)$, such that $f(z)=$ 
$g(w(z))$ (see [15]. In particular, if $g$ is univalent in $U$, then the subordination $f<g$ is equivalent to $f(0)=g(0)$ and $f(U) \subset g(U)$.

A function $f \in A$ is said to be in the class $\mathscr{S}_{\delta}(\gamma)$ if it satisfies

$$
\left(f^{\prime}(z)\right)^{\delta}<\frac{\gamma+z}{\gamma-z}, \quad(z \in U, \gamma>0, \delta>0) .
$$

Also, a function $f \in A$ is said to be in the class $\mathscr{T}_{\delta}(\gamma)$ if it satisfies

$$
\left(\frac{1}{f^{\prime}(z)}\right)^{\delta}<\frac{\gamma+z}{\gamma-z},(z \in U, \gamma>0, \delta>0) .
$$

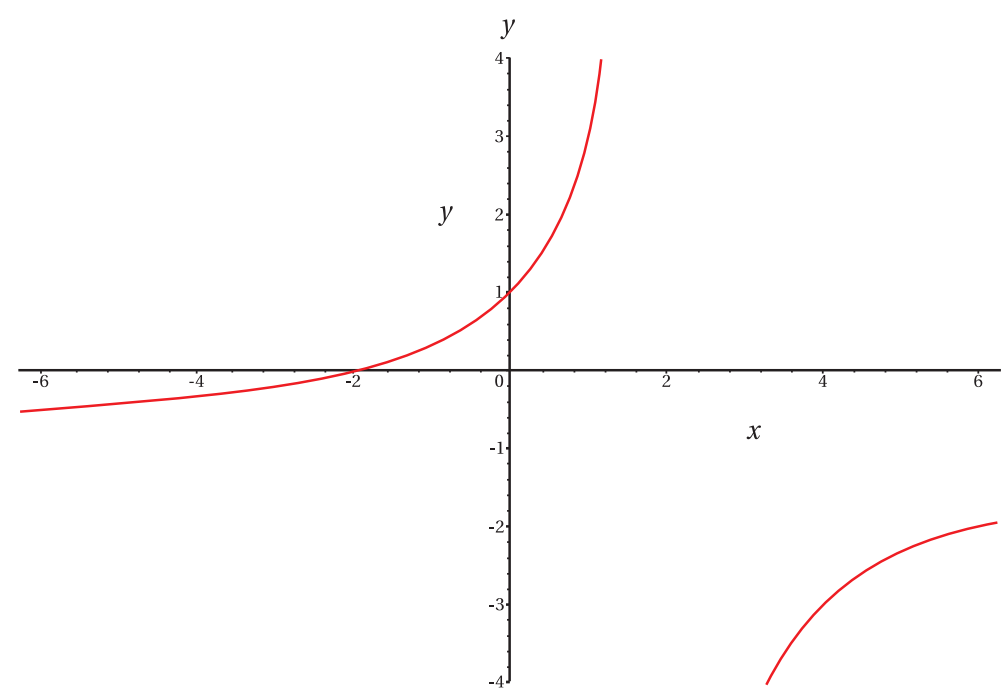

Figure 1.1: $f(z)=\frac{\gamma+z}{\gamma-z}, \gamma>1$.

Now we define a subclass of analytic functions containing the operator (10). Let $f \in \mathscr{A}$ then $f$ is a member of the class $\mathscr{S}_{\mu, \lambda}^{*}(\beta)$ if and only if

$$
\frac{z\left[I_{\mu}^{\lambda}(z, y, x, a) f(z)\right]^{\prime}}{I_{\mu}^{\lambda}(z, y, x, a) f(z)} \prec \frac{1+\beta z}{1-\beta z},(\beta>0) .
$$

To discuss our problem, we recall the following lemma due to Jack [16].

Lemma 1.2. Let $w(z)$ be analytic in $U$ with $w(0)=0$. If $|w(z)|$ attains its maximum value on the circle $|z|=r<1$ at a point $z_{0}$, then

$$
z_{0} w^{\prime}\left(z_{0}\right)=k w\left(z_{0}\right)
$$




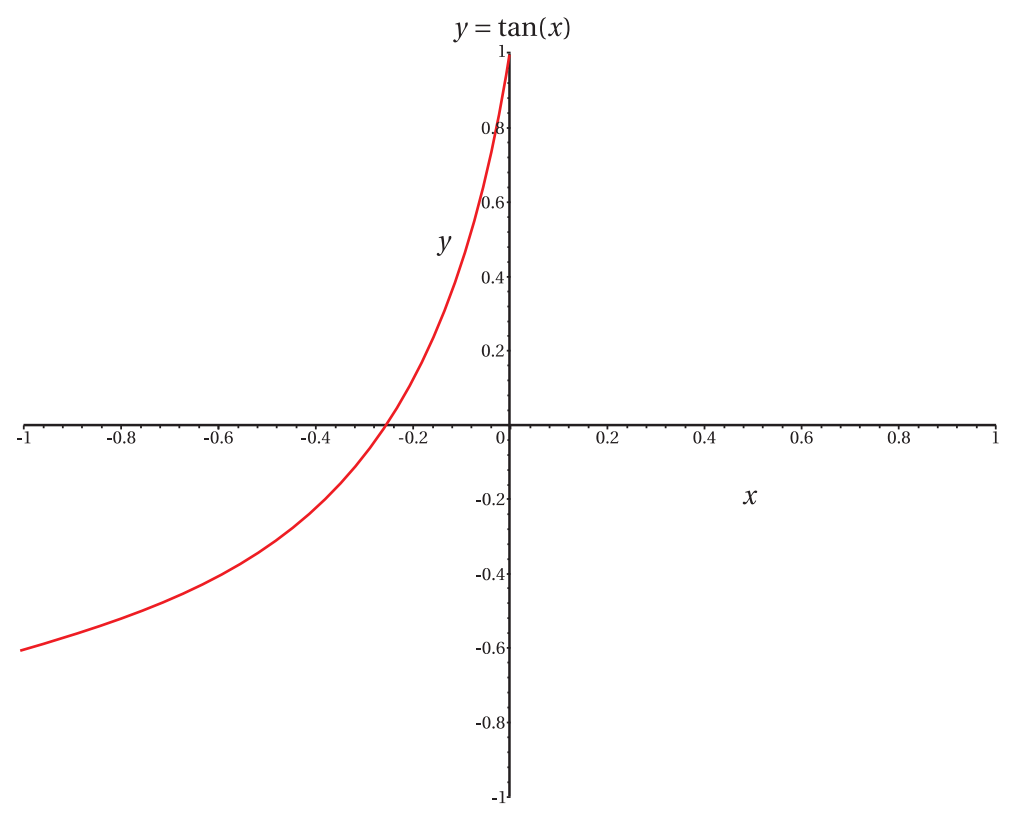

Figure 1.2: $f(z)=\frac{1+\beta z}{1-\beta z}, \beta>0$.

where $k$ is a real number and $k \geq 1$.

Lemma 1.3. (see [17]) Let $\phi$ be convex in $U$ with $\Re\{\kappa \phi(z)+\tau\}>0$ for $\kappa, \tau \in \mathbb{C}$, also let $p(z) \in$ $\mathscr{H}(U)$ with $p(0)=\phi(0)$ and satisfied the Briot-Bouquet differential subordination

$$
p(z)+\frac{z p^{\prime}(z)}{\kappa p(z)+\tau} \prec \phi(z), \quad z \in U
$$

implies that

$$
p(z) \prec \phi(z), z \in U
$$

\section{Main Results}

In this section we establish the sufficient conditions for functions to be in the classes $\mathscr{S}_{\delta}(\gamma), \mathscr{T}_{\delta}(\gamma)$ and $\mathscr{S}_{\mu, \lambda}^{*}(\beta)$. Moreover, we investigate some inclusion properties.

Let us start with the following result:

Theorem 2.1. If $f \in \mathscr{A}$ satisfies

$$
\Re\left(\frac{z f^{\prime \prime}(z)}{f^{\prime}(z)}\right)<\frac{2 \gamma}{\delta(\gamma+1)^{2}}, \quad(z \in U)
$$


for some $\gamma>0, \delta>0$ then $f \in \mathscr{S}_{\delta}(\gamma)$.

Proof. Let $w(z)$ defined by

$$
\left(f^{\prime}(z)\right)^{\delta}=\frac{\gamma+w(z)}{\gamma-w(z)},(\gamma \neq w(z))
$$

Then $w(z)$ is analytic in $U$. From (16) we observe that $w(0)=0$. Also it follows that

$$
\begin{aligned}
& \delta\left(f^{\prime}(z)\right)^{\delta-1} f^{\prime \prime}(z)=\frac{2 \gamma w^{\prime}(z)}{(\gamma-w(z))^{2}} \\
& \Rightarrow \delta\left(f^{\prime}(z)\right)^{\delta} \frac{f^{\prime \prime}(z)}{f^{\prime}(z)}=\frac{2 \gamma w^{\prime}(z)}{(\gamma-w(z))^{2}} \\
& \Rightarrow \delta \frac{f^{\prime \prime}(z)}{f^{\prime}(z)}=\frac{2 \gamma w^{\prime}(z)}{(\gamma-w(z))(\gamma+w(z))} \\
& \Rightarrow \delta \Re\left(\frac{z f^{\prime \prime}(z)}{f^{\prime}(z)}\right)=\Re\left(\frac{2 \gamma z w^{\prime}(z)}{(\gamma-w(z))(\gamma+w(z))}\right)<\frac{2 \gamma}{(\gamma+1)^{2}}, \gamma>0 .
\end{aligned}
$$

Now we proceed to prove that $|w(z)|<1$. Suppose that there exists a point $z_{0} \in U$ such that

$$
\max _{|z| \leq\left|z_{0}\right|}|w(z)|=\left|w\left(z_{0}\right)\right|=1
$$

Then, using the Lemma 1.2 and letting $w\left(z_{0}\right)=e^{i \theta}$ and $z_{0} w^{\prime}\left(z_{0}\right)=k e^{i \theta}, k \geq 1$ yields

$$
\begin{aligned}
\delta \Re\left(\frac{z f^{\prime \prime}\left(z_{0}\right)}{f^{\prime}\left(z_{0}\right)}\right) & =\Re\left(\frac{2 \gamma z_{0} w^{\prime}\left(z_{0}\right)}{\left(\gamma-w\left(z_{0}\right)\right)\left(\gamma+w\left(z_{0}\right)\right)}\right) \\
& =\Re\left(\frac{\left.2 \gamma k e^{i \theta}\right)}{\left(\gamma-e^{i \theta}\right)\left(\gamma+e^{i \theta}\right)}\right) \\
& =\frac{2 k \gamma}{(\gamma+1)^{2}} \geq \frac{2 \gamma}{(\gamma+1)^{2}} .
\end{aligned}
$$

Thus we have

$$
\Re\left(\frac{z f^{\prime \prime}(z)}{f^{\prime}(z)}\right) \geq \frac{2 \gamma}{\delta(\gamma+1)^{2}}, \quad(z \in U)
$$

which contradicts the hypothesis (15). Therefore, we conclude that $|w(z)|<1$ for all $z \in U$ that is

$$
\left(f^{\prime}(z)\right)^{\delta}<\frac{\gamma+z}{\gamma-z},(z \in U, \gamma>0, \delta>0) .
$$

This completes the proof of the theorem.

Corollary 2.2. If $f \in \mathscr{A}$ satisfies the condition (15) for some $0<\gamma<1, \delta>0$, then

$$
\left|\left(f^{\prime}(z)\right)^{\delta}-\frac{1+\gamma}{1-\gamma}\right|<\frac{1+\gamma}{1-\gamma},(z \in U) .
$$


Proof. Since $f$ satisfies the condition (11) implies $f \in \mathscr{S}_{\delta}(\gamma)$, then there exists a function $w(z)$ with $w(0)=0$ and $|w(z)|<1$ such that

$$
\left(f^{\prime}(z)\right)^{\delta}=\frac{\gamma+w(z)}{\gamma-w(z)}
$$

That is

$$
|w(z)|=\left|\frac{\left.\gamma\left(f^{\prime}(z)\right)^{\delta}-1\right)}{\left(f^{\prime}(z)\right)^{\delta}+1}\right|<1
$$

then we obtain (18).

Corollary 2.3. If $f \in \mathscr{A}$ satisfies

$$
\Re\left(\frac{\left(I_{\mu}^{\lambda+1}(z, y, x, a) f(z)\right)^{\prime}}{\left(I_{\mu}^{\lambda}(z, y, x, a) f(z)\right)^{\prime}}\right)-1<\frac{2 \gamma}{\lambda \delta(\gamma+1)^{2}}, \quad(z \in U)
$$

for some $\gamma>1, \delta>0$, then $I_{\mu}^{\lambda}(z, y, x, a) f(z) \in \mathscr{S}_{\delta}(\gamma)$.

Proof. It is clear that for $f \in \mathscr{A}$ we have $I_{\mu}^{\lambda}(z, y, x, a) f(z) \in \mathscr{A}$. By using Lemma 1.1, a computation gives

$$
\begin{aligned}
\Re\left(\frac{z\left(I_{\mu}^{\lambda}(z, y, x, a) f(z)\right)^{\prime \prime}}{\left(I_{\mu}^{\lambda}(z, y, x, a) f(z)\right)^{\prime}}\right) & =\Re\left(\frac{\left(z\left(I_{\mu}^{\lambda}(z, y, x, a) f(z)\right)^{\prime}\right)^{\prime}}{\left(I_{\mu}^{\lambda}(z, y, x, a) f(z)\right)^{\prime}}\right)-1 \\
& =\lambda \Re\left(\frac{\left(I_{\mu}^{\lambda+1}(z, y, x, a) f(z)\right)^{\prime}}{\left(I_{\mu}^{\lambda}(z, y, x, a) f(z)\right)^{\prime}}\right)-\lambda \\
& <\frac{2 \gamma}{\delta(\gamma+1)^{2}},(z \in U) .
\end{aligned}
$$

Hence in view of Theorem 2.1, we obtain $I_{\mu}^{\lambda}(z, y, x, a) f(z) \in \mathscr{S}_{\delta}(\gamma)$.

Theorem 2.4. If $f \in \mathscr{A}$ satisfies

$$
\Re\left(\frac{z f^{\prime \prime}(z)}{f^{\prime}(z)}\right)>\frac{2 \gamma}{\delta\left(1-\gamma^{2}\right)},(z \in U)
$$

for some $\gamma>1, \delta>0$, then $f \in \mathscr{T}_{\delta}(\gamma)$.

Proof. Let $w(z)$ defined by

$$
\left(f^{\prime}(z)\right)^{-\delta}=\frac{\gamma+w(z)}{\gamma-w(z)},(\gamma \neq w(z))
$$


Then $w(z)$ is analytic in $U$. From (20) we observe that $w(0)=0$. Also it follows that

$$
\begin{aligned}
& -\delta\left(f^{\prime}(z)\right)^{-\delta-1} f^{\prime \prime}(z)=\frac{2 \gamma w^{\prime}(z)}{(\gamma-w(z))^{2}} \\
& \Rightarrow-\delta\left(f^{\prime}(z)\right)^{-\delta} \frac{f^{\prime \prime}(z)}{f^{\prime}(z)}=\frac{2 \gamma w^{\prime}(z)}{(\gamma-w(z))^{2}} \\
& \Rightarrow \delta \frac{f^{\prime \prime}(z)}{f^{\prime}(z)}=\frac{2 \gamma w^{\prime}(z)}{(w(z)-\gamma)(\gamma+w(z))} \\
& \Rightarrow \delta \Re\left(\frac{z f^{\prime \prime}(z)}{f^{\prime}(z)}\right)=\Re\left(\frac{2 \gamma z w^{\prime}(z)}{(w(z)-\gamma)(\gamma+w(z))}\right)>\frac{2 \gamma}{1-\gamma^{2}} .
\end{aligned}
$$

Now we proceed to prove that $|w(z)|<1$. Suppose that there exists a point $z_{0} \in U$ such that

$$
\max _{|z| \leq\left|z_{0}\right|}|w(z)|=\left|w\left(z_{0}\right)\right|=1 .
$$

Then, using Lemma 1.2 and letting $w\left(z_{0}\right)=e^{i \theta}$ and $z_{0} w^{\prime}\left(z_{0}\right)=k e^{i \theta}, k \geq 1$ yields

$$
\begin{aligned}
\delta \Re\left(\frac{z f^{\prime \prime}\left(z_{0}\right)}{f^{\prime}\left(z_{0}\right)}\right) & =\Re\left(\frac{2 \gamma z_{0} w^{\prime}\left(z_{0}\right)}{\left(w\left(z_{0}\right)-\gamma\right)\left(\gamma+w\left(z_{0}\right)\right)}\right) \\
& =\Re\left(\frac{\left.2 \gamma k e^{i \theta}\right)}{\left(e^{i \theta}-\gamma\right)\left(\gamma+e^{i \theta}\right)}\right) \\
& =\frac{2 k \gamma}{1-\gamma^{2}} \leq \frac{2 \gamma}{1-\gamma^{2}}, \gamma>1 .
\end{aligned}
$$

Thus we have

$$
\Re\left(\frac{z f^{\prime \prime}(z)}{f^{\prime}(z)}\right) \leq \frac{2 \gamma}{\delta\left(1-\gamma^{2}\right)}, \quad(z \in U)
$$

which contradicts the hypothesis (19). Therefore, we conclude that $|w(z)|<1$ for all $z \in U$ that is

$$
\left(f^{\prime}(z)\right)^{-\delta} \prec \frac{\gamma+z}{\gamma-z}, \quad(z \in U, \gamma>1, \delta>0) .
$$

This completes the proof of the theorem.

In the same manner as in Corollaries 2.2 and 2.3, we have the following result:

Corollary 2.5. If $f \in \mathscr{A}$ satisfies the condition (19) for some $\gamma>1, \delta>0$, then

$$
\left|\left(f^{\prime}(z)\right)^{-\delta}-1\right|<1, \quad(z \in U) .
$$

Corollary 2.6. If $f \in \mathscr{A}$ satisfies

$$
\Re\left(\frac{\left(I_{\mu}^{\lambda+1}(z, y, x, a) f(z)\right)^{\prime}}{\left(I_{\mu}^{\lambda}(z, y, x, a) f(z)\right)^{\prime}}\right)-1>\frac{2 \gamma}{\lambda \delta\left(1-\gamma^{2}\right)}, \quad(z \in U)
$$


for some $\gamma>1, \delta>0$, then $I_{\mu}^{\lambda}(z, y, x, a) f(z) \in \mathscr{T}_{\delta}(\gamma)$.

The following result shows the sufficient condition for functions $f$ to belong to the class $\mathscr{S}_{\mu, \lambda}^{*}(\beta)$.

Theorem 2.7. If $f \in \mathscr{A}$ satisfies

$$
\Re\left(\frac{z\left(I_{\mu}^{\lambda}(z, y, x, a) f(z)\right)^{\prime \prime}}{\left(I_{\mu}^{\lambda}(z, y, x, a) f(z)\right)^{\prime}}+1\right)<\frac{2 \beta}{(\beta+1)^{2}}, \quad(z \in U)
$$

for some $\beta>0$, then $f \in \mathscr{S}_{\mu, \lambda}^{*}(\beta)$.

Proof. Let $w(z)$ defined by

$$
\frac{z\left[I_{\mu}^{\lambda}(z, y, x, a) f(z)\right]^{\prime}}{I_{\mu}^{\lambda}(z, y, x, a) f(z)}=\frac{1+\beta w(z)}{1-\beta w(z)}, \quad(1 \neq \beta w(z)) .
$$

Then $w(z)$ is analytic in $U$. Further, from (24) we have $w(0)=0$. Also it follows that

$$
\begin{gathered}
\frac{z\left(I_{\mu}^{\lambda}(z, y, x, a) f(z)\right)^{\prime \prime}}{\left(I_{\mu}^{\lambda}(z, y, x, a) f(z)\right)^{\prime}}+1=\frac{2 \beta z w^{\prime}(z)}{(1-\beta w(z))^{2}} \\
\Rightarrow \Re\left\{\frac{z\left(I_{\mu}^{\lambda}(z, y, x, a) f(z)\right)^{\prime \prime}}{\left(I_{\mu}^{\lambda}(z, y, x, a) f(z)\right)^{\prime}}+1\right\}=\Re\left\{\frac{2 \beta z w^{\prime}(z)}{(1-\beta w(z))^{2}}\right\} \\
<\frac{2 \beta}{(\beta+1)^{2}}
\end{gathered}
$$

Now we proceed to prove that $|w(z)|<1$. Suppose that there exists a point $z_{0} \in U$ such that

$$
\max _{|z| \leq\left|z_{0}\right|}|w(z)|=\left|w\left(z_{0}\right)\right|=1 .
$$

Then, using Lemma 1.1 and assuming $w\left(z_{0}\right)=e^{i \theta}$ and $z_{0} w^{\prime}\left(z_{0}\right)=k e^{i \theta}, k \geq 1$ yields

$$
\begin{aligned}
\Re\left\{\frac{z_{0}\left(I_{\mu}^{\lambda}(z, y, x, a) f\left(z_{0}\right)\right)^{\prime \prime}}{\left(I_{\mu}^{\lambda}(z, y, x, a) f\left(z_{0}\right)\right)^{\prime}}+1\right\} & =\Re\left\{\frac{2 \beta z_{0} w^{\prime}\left(z_{0}\right)}{\left(1-\beta w_{0}(z)\right)^{2}}\right\} \\
& =\Re\left\{\frac{2 \beta k e^{i \theta}}{\left(1-\beta e^{i \theta}\right)^{2}}\right\} \\
& =\frac{2 k \beta}{(1+\beta)^{2}} \\
& \geq \frac{2 \beta}{(1+\beta)^{2}} .
\end{aligned}
$$

Thus we have

$$
\Re\left(\frac{z\left(I_{\mu}^{\lambda}(z, y, x, a) f(z)\right)^{\prime \prime}}{\left(I_{\mu}^{\lambda}(z, y, x, a) f(z)\right)^{\prime}}+1\right) \geq \frac{2 \beta}{(1+\beta)^{2}}, \quad(z \in U)
$$


which contradicts the hypothesis (23). Therefore, we conclude that $|w(z)|<1$ for all $z \in U$ implies

$$
\frac{z\left[I_{\mu}^{\lambda}(z, y, x, a) f(z)\right]^{\prime}}{I_{\mu}^{\lambda}(z, y, x, a) f(z)} \prec \frac{1+\beta z}{1-\beta z},(\beta>0) .
$$

This completes the proof of the theorem.

Corollary 2.8. If $f \in \mathscr{A}$ satisfies condition (23) for some $0<\beta<1$, then

$$
\left|\frac{z\left[I_{\mu}^{\lambda}(z, y, x, a) f(z)\right]^{\prime}}{I_{\mu}^{\lambda}(z, y, x, a) f(z)}-\frac{1}{1-\beta}\right|<\frac{1}{1-\beta} .
$$

Proof. Since $f$ satisfies the condition (23) implies $f \in \mathscr{S}_{\mu, \lambda}^{*}(\beta)$, then there exists a function $w(z)$ with $w(0)=0$ and $|w(z)|<1$ such that

$$
\frac{z\left[I_{\mu}^{\lambda}(z, y, x, a) f(z)\right]^{\prime}}{I_{\mu}^{\lambda}(z, y, x, a) f(z)}=\frac{1+\beta w(z)}{1-\beta w(z)} .
$$

That is

$$
|w(z)|=\left|\frac{\frac{z\left[I_{\mu}^{\lambda}(z, y, x, a) f(z)\right]^{\prime}}{I_{\mu}^{\lambda}(z, y, x, a) f(z)}-1}{\beta\left[\frac{z\left[I_{\mu}^{\lambda}(z, y, x, a) f(z)\right]^{\prime}}{I_{\mu}^{\lambda}(z, y, x, a) f(z)}+1\right]}\right|<1,
$$

then we obtain (26).

Corollary 2.9. If $f \in \mathscr{A}$ satisfies condition (23) for some $0<\beta<1$, then $I_{\mu}^{\lambda}(z, y, x, a) f(z)$ is starlike in $U$.

Now we introduce the inclusion relations.

Theorem 2.10. Let $\beta>0, \lambda>-1$ and $\mu \in \mathbb{C}$. If $\Re\left\{\frac{I_{\mu}^{\lambda+2}(z, y, x, a) f(z)}{I_{\mu}^{\lambda+1}(z, y, x, a) f(z)}\right\}>\frac{\lambda}{\lambda+1}$, then

$$
\mathscr{S}_{\mu, \lambda}^{*}(\beta) \subset \mathscr{S}_{\mu, \lambda+1}^{*}(\beta) \text {. }
$$

Proof. Our aim is to apply Lemma 1.2. Let $f \in \mathscr{S}_{\mu, \lambda}^{*}(\beta)$ and

$$
p(z)=\frac{z\left[I_{\mu}^{\lambda}(z, y, x, a) f(z)\right]^{\prime}}{I_{\mu}^{\lambda}(z, y, x, a) f(z)},(p(0)=1) .
$$

Then we have

$$
p(z)+\frac{z p^{\prime}(z)}{\kappa p(z)+v} \prec \frac{z\left[I_{\mu}^{\lambda+1}(z, y, x, a) f(z)\right]^{\prime}}{I_{\mu}^{\lambda+1}(z, y, x, a) f(z)}:=\phi(z)
$$


for some $v$ and $\kappa$. It is clear that $\phi(0)=1$. Hence $f \in \mathscr{S}_{\mu, \lambda+1}^{*}(\beta)$.

\section{Conclusion}

From above, we conclude that by using the convolution product (Hadamard product), a linear operator containing the generalized double Hurwitz-Lerch zeta function can be introduced. Some of its properties are discussed and some classes are suggested. One can use the modified Hurwitz-Lerch zeta function as in [12] to define more generalized linear operator.

Moreover, for different classes of analytic functions, for example multivalent functions, meromorphic functions and multivalent meromorphic functions, one can apply (7) to obtain new operators in the unit disc. Other work related to zeta function can be found in $[18,19]$.

\section{Acknowledgement}

The work here is partially supported by UKM-ST-06-FRGS0244-2010.

\section{References}

[1] I. Aleksandar, The Riemann Zeta-Function: Theory and Applications, John Wiley \& Sons, Inc., New York, 1985.

[2] E. Elizalde, Ten Physical Applications of Spectral Zeta Functions, Springer-Verlag, Berlin, 1995.

[3] A. Erdelyi, W. Magnus, F. Oberhettinger, F. G. Tricomi, Higher Transcendental Functions, Vol. I, McGraw-Hill, New York, Toronto and London 1953.

[4] S. Goyal, R. K. Laddha, On the generalized Riemann zeta function and the generalized Lambert transform, Ganita Sandesh, 11 (1997), 99-108.

[5] J. Choi and H. M. Srivastava, Certain families of series associated with the Hurwitz-Lerch zeta function, Appl. Math. Comput., 170 (2005), 399-409.

[6] C. Ferreira, J. L. L'opez, Asymptotic expansions of the Hurwitz-Lerch zeta function, J. Math. Anal. Appl., 298 (2004), 210-224.

[7] M. Garg, P. K. Jain and H. M. Srivastava, Some relationships between the generalized Apostol- Bernoulli polynomials and Hurwitz-Lerch zeta functions, Integral Transforms Spec. Funct., 17 (2006), 803-815.

[8] S. D. Lin and H. M. Srivastava, Some families of the Hurwitz-Lerch zeta functions and associated fractional derivative and other integral representations, Appl. Math. Comput., 154 (2004), 725-733.

[9] S. D. Lin, H. M. Srivastava and P. Y. Wang, Some expansion formulas for a class of generalized Hurwitz-Lerch zeta functions, Integral Transforms Spec. Funct., 17 (2006), 817-827.

[10] Q. M. Luo and H. M. Srivastava, Some generalizations of the Apostol-Bernoulli and Apostol- Euler polynomials, J. Math. Anal. Appl., 308 (2005), 290-302.

[11] E. W. Barnes, The theory of the double gamma function, Philos. Trans. Roy. Soc. A, 196 (1901), 265-387.

[12] M. G. Bin-Saad, Hypergeometric seires assotiated with the Hurwitz-Lerch zeta function, Acta Math. Univ. Comenianae Vol. LXXVIII, 2(2009), 269-286.

[13] B. C. Carlson and D. B. Shaffer, Starlike and prestarlike hypergeometric functions, SIAM J. Math. Anal., 15 (1984), 737-745.

[14] K. I. Noor, Integral operators defined by convolution with hypergeometric functions, Appl. Math. Comp., 182 (2006), 1872-1881.

[15] P. L. Duren, Univalent Functions, Springer-Verlag, New York, Berlin, Heidelberg, Tokyo, 1983. 
[16] I. S. Jack, Functions starlike and convex of order K, J. London Math. Soc., 3(1971), 469-474.

[17] S. S. Miller, P. T. Mocanu, Differential subordinations and univalent functions, Michigan J. Math., 28 (1981), 157-171.

[18] R. W. Ibrahim and M. Darus, Operator defined by convolution with Zeta functions, Far East J. Math Sci., 40(2010), 93-105.

[19] M. Darus, I. Faisal and M. A. M. Nasr, Differential subordination results for some classes of the family $\zeta(\psi, \theta)$ associated with linear operator, Acta Universitatis Sapientiae-Mathematica, 2(2010), 184-194.

School of Mathematical Sciences, Faculty of science and Technology, Universiti Kebangsaan Malaysia, Bangi 43600, Selangor Darul Ehsan, Malaysia.

E-mail: rabhaibrahim@yahoo.com

School of Mathematical Sciences, Faculty of Science and Technology, Universiti Kebangsaan Malaysia, Bangi 43600, Selangor Darul Ehsan, Malaysia.

E-mail: maslina@ukm.my 